\title{
Body weight loss after surgery affects the continuity of adjuvant chemotherapy for pancreatic cancer
}

\author{
Yoshifumi Morita ${ }^{1 *}$ D, Takanori Sakaguchi ${ }^{1}$, Ryo Kitajima', Satoru Furuhashi ${ }^{1}$, Ryota Kiuchi ${ }^{1}$, Makoto Takeda ${ }^{1}$, \\ Takanori Hiraide ${ }^{1}$, Yasushi Shibasaki ${ }^{2}$, Hirotoshi Kikuchi ${ }^{1}$, Hiroyuki Konno ${ }^{3}$ and Hiroya Takeuchi ${ }^{1}$
}

\begin{abstract}
Background: Postoperative chemotherapy is beneficial for many pancreatic cancer patients. However, some patients require dose reduction or the discontinuation of adjuvant chemotherapy because of adverse treatmentrelated effects. In this study, we aimed to evaluate two main outcomes. First, we evaluated the clinicopathological factors affecting patient disease-free survival (DFS) and overall survival (OS) following upfront surgery. Second, we evaluated the factors that influence the continuity of adjuvant chemotherapy.

Methods: Fifty-four patients with resected pancreatic cancer were enrolled. First, we evaluated the clinicopathological factors affecting postoperative survival using the Kaplan-Meier method and Cox regression method. Next, factors affecting the continuity of adjuvant chemotherapy were analyzed using multiple logistic regression analysis.

Results: Univariate and multivariate analyses revealed that positive LN metastasis (HR (95\% Cl) $6.329(2.381-$ 16.95); $p<0.001)$ and relative dose intensity (RDI) < 80\% for adjuvant chemotherapy (HR (95\% Cl) $5.154(1.761-15.15)$; $p=0.003$ ) were independent predictive factors for DFS. Regarding OS, extended dissection of the nerve plexus around the superior mesenteric artery (SMA) (HR ( $95 \% \mathrm{Cl}) 4.504$ (1.721-11.76); $p=0.002)$, positive microscopic surgical margin ( $\mathrm{HR}(95 \% \mathrm{Cl}) 5.565$ (1.724-17.96); $p=0.004)$, and adjuvant chemotherapy of RDI < 80\% (HR (95\% Cl) 3.534 (1.135-2.667); $p=0.029$ ) were also independent predictive factors. Moreover, the level of RDI significantly correlated with DFS and OS. Multiple logistic regression analysis revealed that low RDI was significantly associated with postoperative body weight loss (BWL) $\geq 10 \%$.

Conclusions: The following factors were significantly associated with poor survival: extended dissection of the nerve plexus around the SMA, lymph node metastasis, residual tumor, and RDI of the adjuvant chemotherapy. Patient's prognosis with adjuvant chemotherapy of $\mathrm{RDI}<80 \%$ was worse. BWL $\geq 10 \%$ was the most important factor affecting the continuity of adjuvant chemotherapy. Perioperative nutritional intervention is necessary for patients who receive adjuvant chemotherapy for advanced pancreatic cancer.
\end{abstract}

Keywords: Adjuvant chemotherapy, Body weight loss, Continuity, Pancreatic cancer

\footnotetext{
* Correspondence: yoshi-mo@hama-med.ac.jp

${ }^{1}$ Second Department of Surgery, Hamamatsu University School of Medicine,

1-20-1 Handayama, Higashi-ku, Hamamatsu 431-3192, Japan

Full list of author information is available at the end of the article
}

(c) The Author(s). 2019 Open Access This article is distributed under the terms of the Creative Commons Attribution 4.0 International License (http://creativecommons.org/licenses/by/4.0/), which permits unrestricted use, distribution, and reproduction in any medium, provided you give appropriate credit to the original author(s) and the source, provide a link to the Creative Commons license, and indicate if changes were made. The Creative Commons Public Domain Dedication waiver (http://creativecommons.org/publicdomain/zero/1.0/) applies to the data made available in this article, unless otherwise stated. 


\section{Background}

Pancreatic cancer is the seventh leading cause of cancer death with more than 450,000 newly diagnosed per year worldwide with increasing rates seen in Europe, North America and Eastern Asian countries [1]. Recent data showed that over the past 10 years, the number of pancreatic cancer patients have been rising at an average annual rate of $0.5 \%$ [2]. In the United States, pancreatic cancer is the fourth-leading cause of cancer death with an estimated 55,440 new cases and 44,330 deaths in 2018 [3]. The 5-year survival for pancreatic cancer is the lowest among various cancers, despite recent advances in understanding its biology and improvements in imaging [4]. At present, surgical resection is the only potentially curative approach for pancreatic cancer when the disease is localized. Upfront surgery followed by adjuvant chemotherapy is recommended in patients with potentially resectable pancreatic cancer by the European Society for Medical Oncology (ESMO) and National Comprehensive Cancer Network (NCCN) guidelines $[5,6]$. However, even after curative resection, most patients experience recurrence within 2 years and $2 / 3$ patients die within 3 years [7]. Meanwhile, patients with borderline resectable cancer should be considered for neoadjuvant chemotherapy. Although the best regimens in the neoadjuvant setting are still undetermined, FOLFIRINOX or Nab-paclitaxel are promising in patients with borderline resectable or locally advanced disease $[8,9]$.

Adjuvant treatment with gemcitabine had been a gold-standard chemotherapeutic agent for pancreatic cancer [10, 11]. The Japan Adjuvant Study Group of Pancreatic Cancer (JASPAC-01) phase III trial demonstrated that S-1 was more effective for pancreatic cancer than gemcitabine [12]. Recently, the European Study Group for Pancreatic Cancer (ESPAC-4) reported that the combination of gemcitabine and capecitabine was superior to gemcitabine monotherapy in patients with resected pancreatic cancer [13].

Continuity of treatment and maintenance of the dose intensity are important for maximizing the efficacy of adjuvant chemotherapy. However, because of various side effects, reduction in the dose intensity or discontinuation of chemotherapy is sometimes required. Therefore, predicting the therapeutic course of such patients represents a major challenge to providing appropriate disease management. In this retrospective analysis of patients that underwent upfront surgery for pancreatic cancer, we aimed to evaluate two main outcomes. First, we evaluated the clinicopathological factors affecting patient disease-free survival (DFS) and overall survival (OS) following pancreatic resection. Second, we evaluated the factors that influence the continuity of post-surgical adjuvant chemotherapy.

\section{Methods}

\section{Patients}

From 2006 to 2016, 107 patients with pancreatic tumors underwent surgical resection at the Hamamatsu University School of Medicine. Seventy-three of 107 patients were diagnosed as having pancreatic cancer. Of these 73 patients, 19 were excluded from the analysis for the following reasons: 10 had an early relapse of less than 6 months, 4 received neoadjuvant chemotherapy, 3 were lost to follow up, 1 patient had stage 0 disease, and 1 patient had multiple primary malignancies. In total, 54 patients were enrolled in this study (Fig. 1).

TNM stage was assessed according to the 7th edition TNM staging guidelines, published by the American Joint Committee on Cancer [14]. The study protocol conformed to the ethical guidelines of the 1975 Declaration of Helsinki and was approval by the ethical committee of our institution (approval number: 15-318). Written consents to participate in this study were substituted for providing a means to opt out in the website (https://www.hama-med.ac.jp/research/clinicalres/erc/disclosure-info/h29.html) according to the ethics guidelines for clinical studies of the Japanese Ministry of Health, Labour and Welfare (MHLW).

\section{Outcome}

We evaluated two main outcomes. Our primary outcome evaluated the clinicopathological factors affecting patient DFS and OS. DFS was calculated as the duration between the operative day and recurrence. Tumor recurrence was determined by CT, cytology or biopsy. OS was calculated as the duration between the operative day and cancer-related death. The secondary outcome examined

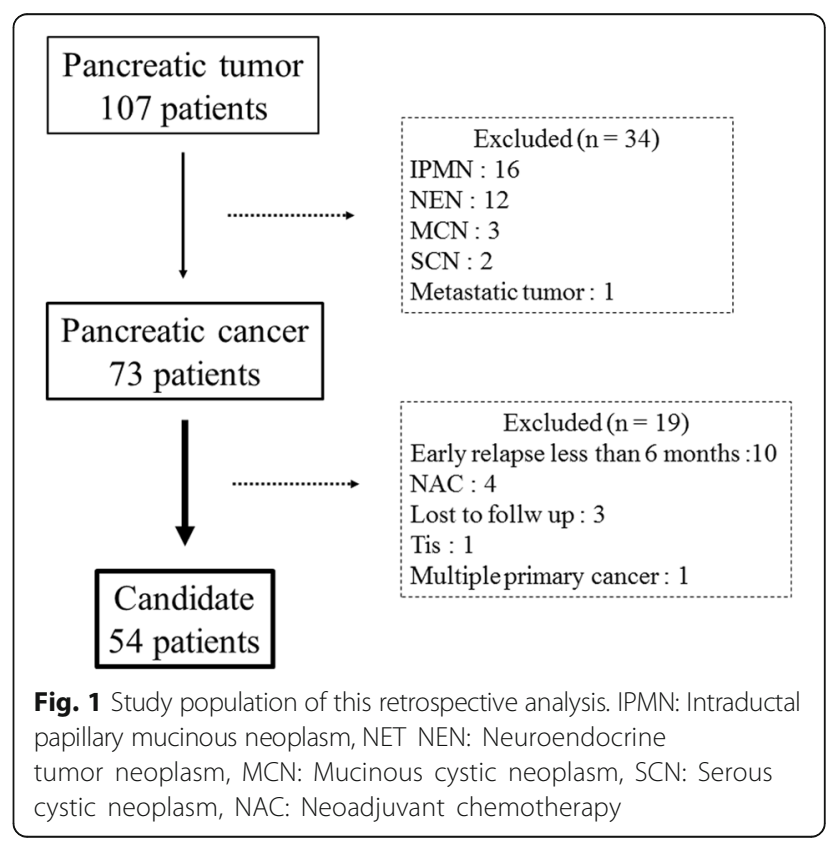


the influential factors affecting the continuity of adjuvant chemotherapy including age, operative methods, postoperative complications, blood test results, and body weight loss (BWL). Statistical methods are described in the following section.

\section{Surgical procedures}

Lymphadenectomy for pancreatic head cancer included the anterior and posterior pancreaticoduodenal, pyloric region, hepatoduodenal ligament, common hepatic artery, and superior and inferior pancreatic head lymph nodes. Lymphadenectomy for pancreatic body and tail cancer included celiac trunk, splenic artery, common hepatic artery, splenic hilum, and superior and inferior pancreatic body lymph nodes. Dissection of the nerve plexus around the superior mesenteric artery (SMA) was performed according to the location and extent of the tumor. Concomitant superior mesenteric vein (SMV) and portal vein resection was performed when the involvement of the SMV and portal vein could be safely reconstructed by direct suture or venous graft.

\section{Postoperative chemotherapy}

For the postoperative adjuvant chemotherapy regimen, patients received either gemcitabine or tegafur/gimeracil/oteracil (S-1). For 5 patients, the treatment was changed from either gemcitabine to S-1 or S-1 to gemcitabine. One treatment cycle consisted of weekly intravenous infusions of $1000 \mathrm{mg} / \mathrm{m}^{2}$ of gemcitabine for $1 \mathrm{~h}$, for 3 weeks, followed by a 1 -week break. S-1 was administered orally at a dose of $80-120 \mathrm{mg}$ per body surface area (BSA) per day (BSA $<1.25 \mathrm{~m}^{2}, 80 \mathrm{mg} /$ day; 1.25 $<$ BSA $<1.5 \mathrm{~m}^{2}, 100 \mathrm{mg} /$ day; $1.5 \mathrm{~m}^{2}>\mathrm{BSA}, 120 \mathrm{mg}$ /day). Each chemotherapy cycle consisted of S-1 administration for 28 days, followed by 14 days without treatment. For both treatment regimens, the planned treatment period was 6 cycles. Relative dose intensity (RDI) was estimated to be $100 \%$ for gemcitabine when the total dose of $18,000 \mathrm{mg} / \mathrm{m}^{2}$ was reached. For S-1, RDI was estimated as $100 \%$ when the total S-1 dose of 13,440 $20,160 \mathrm{mg}$, according to BSA (BSA $<1.25 \mathrm{~m}^{2}, 13,440 \mathrm{mg}$; $1.25<\mathrm{BSA}<1.5 \mathrm{~m}^{2}, 16,800 \mathrm{mg} ; 1.5 \mathrm{~m}^{2}>\mathrm{BSA}, 20160$ $\mathrm{mg}$ ), was achieved. When adverse reactions occurred, either the dose was reduced, the dosing interval was adjusted, or administration was temporarily discontinued. Treatment was discontinued or switched to another regimen when the patient showed disease recurrence or adverse events that were uncontrollable by dose modification or temporary cessation of treatment. Adverse events were recorded according to the Common Terminology Criteria for Adverse Events verssion 4.0 [15].

\section{Statistical analysis}

Body weight loss (BWL) was defined as follows:
Percent $\mathrm{BWL}=$ (preoperative body weight - body weight at the time of discharge) $\times 100 /$ preoperative body weight. Preoperative body weight was measured 23 days before surgery. Renal function was measured in terms of creatinine clearance $(\mathrm{CCr})$, calculated according to the formula proposed by Cockroft and Gault [16]. Variables were compared using the $\chi^{2}$ test, Fisher's exact test, or Mann-Whitney's U test, as appropriate. The cumulative DFS and OS rates were estimated using the Kaplan-Meier method and compared according to the log-rank test. Predictors of outcome were assessed by univariate and multivariate analysis using Cox proportional hazard regression modeling. The stepwise variable selection process was used in the multivariate analysis to identify the most concise model for predicting cumulative survival. Spearman's rank-correlation coefficient was performed to identify correlation between RDI of adjuvant chemotherapy and DFS and OS. Multivariate logistic regression analysis was performed to identify factors that affect the continuity of adjuvant chemotherapy. A $P$ value $<0.05$ was defined as statistically significant. SPSS version 24 (IBM Corp., Armonk, NY, USA) was used for all statistical analyses.

\section{Results}

\section{Clinicopathological characteristics}

Clinicopathological characteristics of the entire cohort, as stratified by treatment group, are presented in Table 1 . The mean age at diagnosis was 70.4 years (range 45-85 years) with the cohort consisting of 26 males and 28 females. Thirty-nine patients $(67.2 \%)$ received adjuvant chemotherapy. Eleven patients tolerated an RDI of $\geq 80 \%$, although the mean RDIs of S-1 and gemcitabine in the adjuvant chemotherapy group were 71.4 and $70.5 \%$, respectively. Fifteen patients $(27.8 \%)$ did not receive adjuvant therapy after surgery, due to their overall general condition, the presence of comorbidities, or at their own discretion. The RDI for these 15 patients was set to 0 . The following clinicopathological characteristics were similar between the 2 groups: surgical method, comorbidities, preoperative BMI, duration of surgery, intraoperative bleeding, number of resected lymph nodes, TNM stage, residual tumor, postoperative diarrhea, clinically-relevant pancreatic fistula and delayed gastric emptying.

\section{Disease-free survival and overall survival}

The median follow-up time of all patients was 30 months (range 5-111 months). At the time of analysis, $24(44.4 \%)$ of the study patients had died. The median DFS for the entire cohort was 30 months. In the univariate analysis, variables such as extended dissection of the nerve plexus around the SMA (17 months versus 52 months; $p=0.007)$, positive lymph node $(\mathrm{LN})$ metastasis 
Table 1 Patient clinicopathological characteristics with or without Adjuvant chemotherapy

\begin{tabular}{|c|c|c|c|}
\hline & Adjuvant (+) N=39 & Adjuvant $(-) N=15$ & $p$ \\
\hline Age $($ mean $\pm S D)$ & $69.9 \pm 7.8$ & $71.5 \pm 7.7$ & 0.499 \\
\hline Sex (Male: Female) & $17: 22$ & 9: 6 & 0.366 \\
\hline Operative method (PD: DP) & 28: 11 & $12: 3$ & 0.733 \\
\hline Comorbidity (Y: N) & 27: 12 & 12: 3 & 0.515 \\
\hline Preoperative $\mathrm{BMI}\left(\mathrm{Kg} / \mathrm{m}^{2}\right)$ (mean $\left.\pm \mathrm{SD}\right)$ & $22.3 \pm 2.9$ & $22.4 \pm 3.4$ & 0.863 \\
\hline Operation time (min) (mean \pm SD) & $396 \pm 100$ & $394 \pm 70$ & 0.955 \\
\hline Intraoperative bleeding $(\mathrm{ml})($ mean $\pm \mathrm{SD})$ & $878 \pm 626$ & $1102 \pm 1339$ & 0.404 \\
\hline Extended dissection of nerve plexus around SMA $(\mathrm{Y}: \mathrm{N})^{\mathrm{a}}$ & 12: 27 & 3: 12 & 0.515 \\
\hline Concomitant venous reconstruction ( $\mathrm{Y}: \mathrm{N})$ & 5: 34 & 1: 14 & 0.461 \\
\hline Number of resected lymph nodes & $30 \pm 11$ & $28 \pm 20$ & 0.730 \\
\hline AJCC Stage (IA: IB: \|A: IIB: III: IV) & 2:0:10:23:0:4 & $3: 2: 3: 7: 0: 0$ & 0.124 \\
\hline Residual tumor (R0: R1) & $32: 7$ & 15: 0 & 0.171 \\
\hline Postoperative diarrhea $(\mathrm{Y}: \mathrm{N})^{\mathrm{b}}$ & 13: 26 & $7: 8$ & 0.363 \\
\hline Postoperative pancreatic fistula grade B or C & 11: 28 & 1: 14 & 0.145 \\
\hline Postoperative delayed gastric emptying (Y: N) & $7: 32$ & 2: 13 & 0.697 \\
\hline
\end{tabular}

${ }^{a}$ Extended dissection of nerve plexus around SMA was defined as more than a half

${ }^{\mathrm{b}}$ Postoperative diarrhea was defined as increased frequency of defecation one or more times per day

(21 months versus not reached; $p=0.005$ ), AJCC Stage (20 months versus not reached; $p=0.010)$, and reduced RDI of the adjuvant chemotherapy $(<80 \%)$ (23 months versus not reached; $p=0.036)$ were significantly associated with poor DFS (Table 2). In the multivariate analysis, positive LN metastasis (HR (95\% CI) 6.329 (2.381$16.95) ; p<0.001$ ), and an RDI $<80 \%$ for the adjuvant chemotherapy (HR (95\% CI) 5.154 (1.761-15.15); $p=$ $0.003)$ were independent predictive factors for DFS (Table 2). This analysis included both adjuvant $(+)$ and adjuvant $(-)$ patients; therefore, for the adjuvant $(+)$ patients, DFS was compared between patients with an RDI $\geq 80 \%$ and those with an RDI $<80 \%$. As shown in Fig. 2a, patients receiving a higher RDI showed significantly better DFS $(p=0.032)$.

Regarding OS, variables such as extended dissection of the nerve plexus around the SMA (22 months versus 67 months; $p=0.011$ ), AJCC Stage (28 months versus not reached; $p=0.026$ ), and reduced RDI of the adjuvant chemotherapy (46 months versus not reached; $p=0.045$ ) were significantly worse in the univariate analysis (Table 3 ). In the multivariate analysis, extended dissection of the nerve plexus around the SMA (HR (95\% CI) 4.504 (1.72111.76); $p=0.002$ ), positive microscopic surgical margin (R1) (HR (95\% CI) 5.565 (1.724-17.96); $p=0.004)$, and adjuvant chemotherapy of RDI < 80\% (HR (95\% CI) 3.534 (1.1352.667); $p=0.029$ ) were also independent predictive factors for poor prognosis (Table 3). Similar to trend for DFS, patients who received $\mathrm{RDI} \geq 80 \%$ tended to have longer OS than those with an RDI $<80 \%(p=0.086)$ (Fig. 2b). Moreover, the level of RDI significantly correlated with DFS and OS for the adjuvant $(+)$ patients, respectively (Fig. 3).
In the total cohort of 54 patients with surgically resected pancreatic cancer, BWL $\geq 10 \%$ tended to be related with worse DFS (26 months versus not reached; $p=$ 0.218 ) and OS (46 months versus not reached; $p=0.163$ ), respectively.

\section{Clinicopathological factors affecting adjuvant chemotherapy continuity}

To determine factors that influence adjuvant chemotherapy continuity, 39 patients who received adjuvant chemotherapy were stratified according to RDI. Detailed clinicopathological characteristics of patients receiving adjuvant chemotherapy are presented in Table 4. Variables associated with RDI $<80 \%$ were age $\geq 75$ years and BWL $\geq 10 \%$. The incidence of postoperative diarrhea, clinically-relevant pancreatic fistula, and delayed gastric emptying were similar between the 2 groups. Multiple logistic regression analysis revealed $\mathrm{BWL} \geq 10 \%$ as an independent predictable factor of adjuvant chemotherapy continuity (Table 5). Importantly, the severity of adverse events during adjuvant chemotherapy was similar between patients with $\mathrm{BWL} \geq 10 \%$ and $\mathrm{BWL}<10 \%$. However, adjuvant chemotherapy discontinuation rate was significantly higher in patients with BWL $\geq 10 \%$ (50\% vs 9.5\%) (Table 6).

\section{Discussion}

Pancreatic cancer is one of most challenging malignancies despite developments in surgery, chemotherapy and radiation therapy. Previously, numerous clinicopathological factors such as lymph node metastasis, portal vein invasion, surgical margin, and postoperative CA19-9 
Table 2 Univariate and multivariate analyses for disease free survival

\begin{tabular}{|c|c|c|c|c|c|}
\hline \multirow[t]{3}{*}{ Variable } & \multirow{2}{*}{\multicolumn{3}{|c|}{$\begin{array}{l}\text { Univariate } \\
\text { Median }\end{array}$}} & \multicolumn{2}{|l|}{ Multivariate } \\
\hline & & & & \multirow[b]{2}{*}{ HR $(95 \%$ Cl) } & \multirow[b]{2}{*}{$p$-Value } \\
\hline & No. & survival & $p$-Value & & \\
\hline \multicolumn{6}{|l|}{$\mathrm{BWL}^{\mathrm{a}}$} \\
\hline$<10 \%$ & 28 & N.R. & & & \\
\hline$\geq 10 \%$ & 26 & 26 & 0.218 & & N.S. \\
\hline \multicolumn{6}{|l|}{ Postoperative diarrhea } \\
\hline No & 34 & 40 & & & \\
\hline Yes & 20 & 21 & 0.194 & & N.S. \\
\hline \multicolumn{6}{|c|}{ Extended dissection of nerve plexus around SMA ${ }^{b}$} \\
\hline No & 39 & 52 & & & \\
\hline Yes & 15 & 17 & 0.007 & & N.S. \\
\hline \multicolumn{6}{|l|}{ Residual tumor } \\
\hline Ro & 47 & 40 & & & \\
\hline R1 & 7 & 12 & 0.144 & & N.S. \\
\hline \multicolumn{6}{|l|}{ Lymph node metastasis } \\
\hline Negative & 20 & N.R. & & & \\
\hline Positive & 34 & 21 & 0.005 & $3.636(1.266-9.524)$ & 0.009 \\
\hline \multicolumn{6}{|l|}{ AJCC Stage } \\
\hline Stage IA, IB & 7 & N.R. & & & \\
\hline Stage IIA or higher & 47 & 20 & 0.010 & & N.S. \\
\hline \multicolumn{6}{|c|}{$\mathrm{RDI}^{\mathrm{a}}$ in adjuvant chemotherapy } \\
\hline$\geq 80 \%$ & 11 & N.R. & & & \\
\hline$<80 \%$ & 43 & 23 & 0.036 & $5.421(1.852-15.86)$ & 0.002 \\
\hline
\end{tabular}

${ }_{\mathrm{a}}^{\mathrm{BWL}}$ (body weight loss): $\%$ body weight loss $=$ (preoperative body weight - body weight at the time of discharge) $\times 100 /$ preoperative body weight ${ }^{b}$ Extended dissection of nerve plexus around SMA was defined as more than a half

$R D I$ Relative dose intensity, N.R. not reached

Boldface indicates the statistical significance

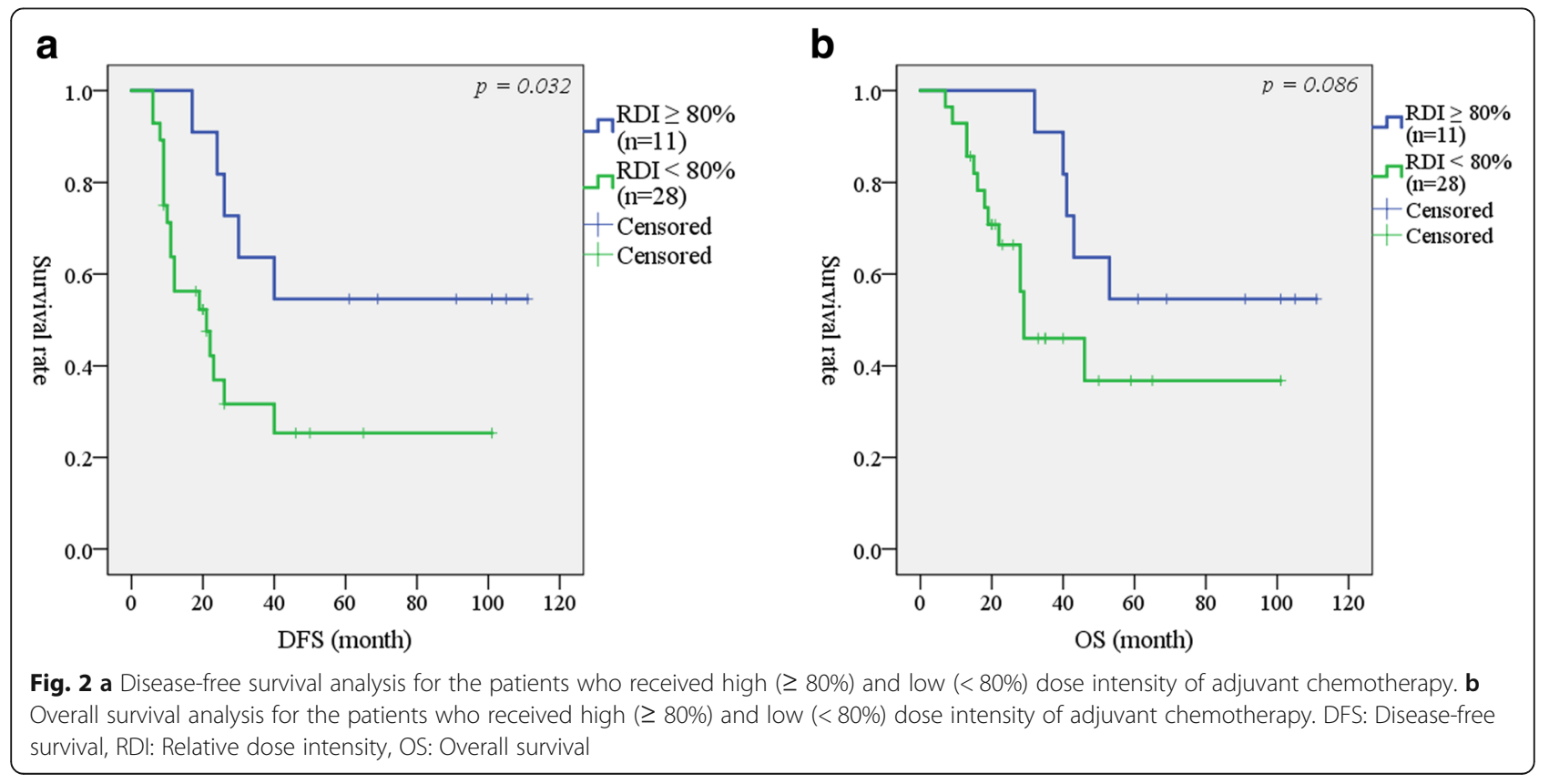


Table 3 Univariate and multivariate analyses for overall survival

\begin{tabular}{|c|c|c|c|c|c|}
\hline \multirow[b]{3}{*}{ Variable } & \multirow[b]{3}{*}{ No. } & \multirow{2}{*}{\multicolumn{2}{|c|}{$\begin{array}{l}\text { Univariate } \\
\text { Median }\end{array}$}} & \multicolumn{2}{|l|}{ Multivariate } \\
\hline & & & & \multirow[b]{2}{*}{ HR (95\% Cl) } & \multirow[b]{2}{*}{$p$-Value } \\
\hline & & survival & $p$-Value & & \\
\hline \multicolumn{6}{|l|}{$\mathrm{BWL}^{\mathrm{a}}$} \\
\hline$<10 \%$ & 28 & N.R. & & & \\
\hline$\geq 10 \%$ & 26 & 46 & 0.163 & & N.S. \\
\hline \multicolumn{6}{|c|}{ Postoperative diarrhea } \\
\hline No & 34 & 40 & & & \\
\hline Yes & 20 & 21 & 0.379 & & N.S. \\
\hline
\end{tabular}

Extended dissection of nerve plexus around SMA ${ }^{\mathrm{b}}$

$\begin{array}{lll}\text { No } & 39 & 67 \\ \text { Yes } & 15 & 22\end{array}$

Residual tumor

$\begin{array}{lll}\text { Ro } & 47 & 53 \\ \text { R1 } & 7 & 28\end{array}$

R1

Lymph node metastasis

$\begin{array}{lll}\text { Negative } & 20 & 67 \\ \text { Positive } & 34 & 40\end{array}$

Positive

AJCC Stage

Stage IA, IB

Stage IIA or higher

RDI in adjuvant chemotherapy

$$
\begin{array}{ll}
\geq 80 \% & 11 \\
<80 \% & 43
\end{array}
$$

${ }^{\mathrm{a} B W L}$ (body weight loss): $\%$ body weight loss $=$ (preoperative body weight - body weight at the time of discharge) $\times 100 /$ preoperative body weight ${ }^{\mathrm{b}}$ Extended dissection of nerve plexus around SMA was defined as more than a half

$R D I$ Relative dose intensity, N.R. not reached

Boldface indicates the statistical significance
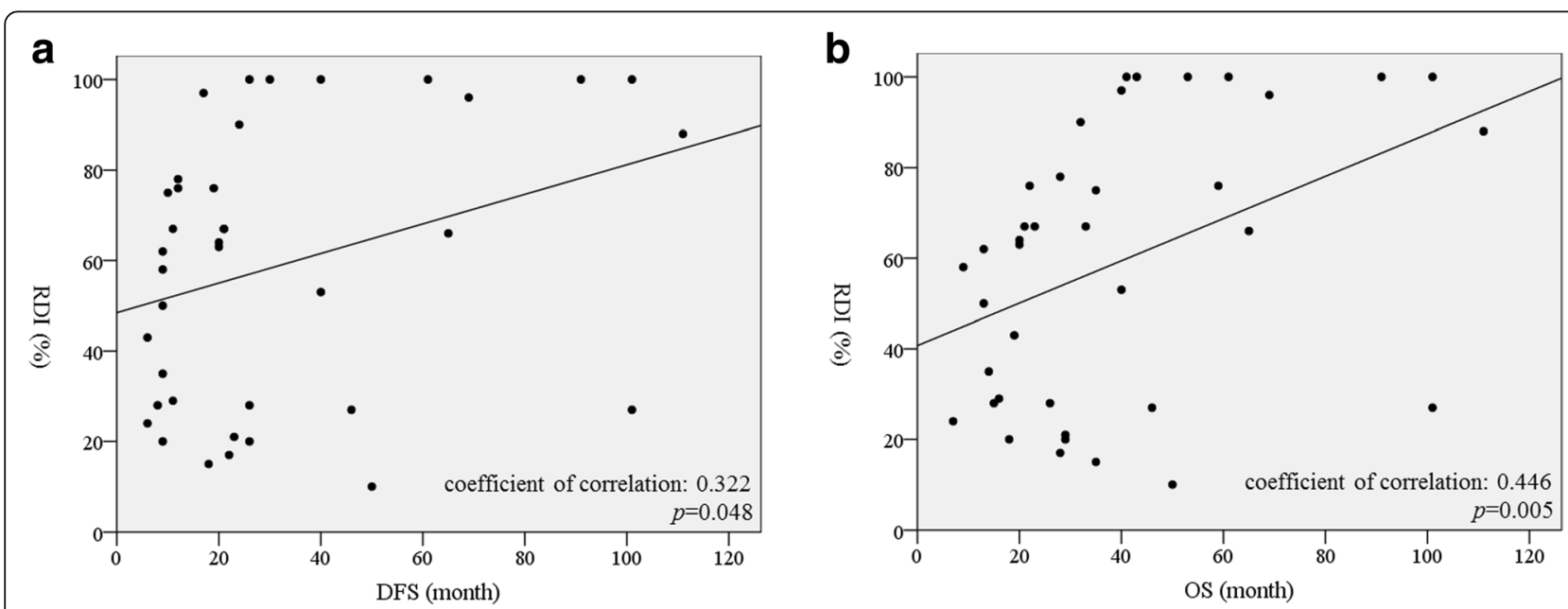

Fig. 3 a Correlation between the level of relative dose intensity and disease-free survivals. b Correlation between the level of relative dose intensity and overall survivals. Black circle indicates each patient with adjuvant chemotherapy. DFS: Disease-free survival, RDI: Relative dose intensity, OS: Overall survival 
Table 4 Clinicopathological characteristics of patients with adjuvant chemotherapy

\begin{tabular}{|c|c|c|c|}
\hline & $\mathrm{RDI} \geq 80 \%$ & $\mathrm{RDI}<80 \%$ & $p$ \\
\hline & $N=11$ & $N=28$ & \\
\hline Age $($ mean $\pm S D)$ & $63.4 \pm 8.0$ & $72.5 \pm 6.1$ & $<0.001$ \\
\hline Age $\geq 75(Y: N)$ & 0: 11 & 11: 17 & 0.017 \\
\hline Sex (Male: Female) & 5: 6 & 12: 16 & N.S. \\
\hline Comorbidity (Y: N) & $6: 5$ & 21: 7 & N.S. \\
\hline Operative method (PD: DP) & $7: 4$ & $21: 7$ & N.S. \\
\hline Extended dissection of nerve plexus around SMA $(Y: N)^{a}$ & 2: 9 & 10: 18 & N.S. \\
\hline Postoperative diarrhea (Y: N) & 3: 8 & 10: 18 & N.S. \\
\hline Postoperative pancreatic fistula grade B or $C$ & 3: 8 & 8: 20 & N.S. \\
\hline Postoperative delayed gastric emptying (Y: N) & $3: 8$ & 4: 24 & N.S. \\
\hline Regimen (GEM: S-1: Both) & $3: 6: 2$ & 5: $20: 3$ & N.S. \\
\hline Postoperative blood test & & & \\
\hline WBC $(/ \mu l)$ & $5238 \pm 1588$ & $5784 \pm 1376$ & N.S. \\
\hline Neutrophil & $3528 \pm 1333$ & $3860 \pm 1135$ & N.S. \\
\hline $\mathrm{Hg}(\mathrm{g} / \mathrm{dl})$ & $11.2 \pm 1.4$ & $10.7 \pm 1.1$ & N.S. \\
\hline $\operatorname{PLT}\left({ }^{*} 10^{4} / \mu \mathrm{l}\right)$ & $35.5 \pm 11.7$ & $32.5 \pm 12.5$ & N.S. \\
\hline AST (IU/I) & $43.2 \pm 23.0$ & $35.1 \pm 19.4$ & N.S. \\
\hline $\mathrm{ALT}(\mathrm{IU} / \mathrm{I})$ & $65.9 \pm 57.8$ & $39.1 \pm 30.0$ & N.S. \\
\hline BUN (mg/dl) & $12.6 \pm 6.1$ & $11.1 \pm 3.1$ & N.S. \\
\hline Cre $(\mathrm{mg} / \mathrm{dl})$ & $0.65 \pm 0.24$ & $0.67 \pm 0.20$ & N.S. \\
\hline $\mathrm{CCr}(\mathrm{ml} / \mathrm{min})$ & $75.2 \pm 25.1$ & $62.2 \pm 17.5$ & N.S. \\
\hline Albumin $(g / d l)$ & $3.4 \pm 0.3$ & $3.1 \pm 0.4$ & N.S. \\
\hline Albumin maintenance rate $\geq 90 \%(\mathrm{Y}: \mathrm{N})^{\mathrm{b}}$ & 3: 8 & $6: 22$ & N.S. \\
\hline$B W L \geq 10 \%(Y: N)^{c}$ & $2: 9$ & 16: 12 & 0.037 \\
\hline
\end{tabular}

${ }^{a}$ Extended dissection of nerve plexus around SMA was defined as more than a half

${ }^{b}$ Albumin maintenance rate was defined as: $\%$ albumin maintenance $=$ albumin value at the time of discharge $/$ preoperative albumin value $\times 100$

${ }^{c}$ BWL (body weight loss): $\%$ body weight loss $=$ (preoperative body weight - body weight at the time of discharge) $\times 100 /$ preoperative body weight

Boldface indicates the statistical significance

level have been reported as prognostic indicators in patients with resected pancreatic cancer [17-20]. In this retrospective analysis, we identified factors including extended dissection of the nerve plexus around the SMA, lymph node metastasis, residual tumor, and adjuvant chemotherapy of RDI $<80 \%$ as prognostic indicator. Additionally, we identified BWL after surgery

Table 5 Multiple logistic regression analysis for continuity of adjuvant chemotherapy

\begin{tabular}{llll}
\hline Variable & No. & OR $(95 \% \mathrm{Cl})$ & -Value \\
\hline Age & & & \\
$<75$ & 28 & & \\
$\geq 75$ & 11 & & \\
BWL $(\%)^{a}$ & & & \\
$<10$ & 21 & $0.167(0.030-0.910)$ & $\mathbf{0 . 0 3 9}$ \\
$\geq 10 \%$ & 18 & & \\
\hline
\end{tabular}

${ }^{\mathrm{a}} \mathrm{BWL}$ (body weight loss): $\%$ body weight loss = (preoperative body weight - body weight at the time of discharge) $\times 100 /$ preoperative body weight

Boldface indicates the statistical significance as an influential factor of the continuity of adjuvant chemotherapy.

The CONKO-001 randomized trial compared postoperative prognosis between pancreatic cancer patients receiving adjuvant gemcitabine and those receiving surgery alone $[10,11]$; the above study confirmed the benefit of gemcitabine-based adjuvant chemotherapy. Recently, the JASPAC-01 study showed that S-1 was superior to gemcitabine for both recurrence-free and overall survival [12]. More recently, the ESPAC-4 phase III trial showed the benefit of adjuvant chemotherapy using gemcitabine combined with capecitabine. While adjuvant chemotherapy has been widely accepted to improve patient prognosis, the dose intensity must be reduced or the chemotherapy must be discontinued because of various side effects. Therefore, it is important to identify risk factors that affect adjuvant chemotherapy continuity to improve the patient survival.

In gemcitabine-based adjuvant treatment, an inflammationbased prognostic score, the Glasgow Prognostic Score 
Table 6 Adverse events during adjuvant chemotherapy

\begin{tabular}{|c|c|c|}
\hline $\mathrm{BWL}<10 \%$ & $\mathrm{BWL} \geq 10 \%$ & $p$ \\
\hline$N=21$ & $N=18$ & \\
\hline \multicolumn{3}{|c|}{ (Number: CTCAE Grade 4, 3, 2, 1) } \\
\hline \multicolumn{3}{|l|}{ Anemia } \\
\hline$(0,0,1,0)$ & $(0,0,2,0)$ & \\
\hline \multicolumn{3}{|l|}{ Leucopenia } \\
\hline$(0,1,2,0)$ & $(0,1,3,0)$ & \\
\hline \multicolumn{3}{|l|}{ Neutropenia } \\
\hline$(0,3,2,1)$ & $(1,1,1,0)$ & \\
\hline \multicolumn{3}{|c|}{ Thrombopenia } \\
\hline$(0,0,1,0)$ & $(0,0,0,0)$ & \\
\hline \multicolumn{3}{|c|}{ Hepatic toxicity } \\
\hline$(0,1,3,2)$ & $(0,0,0,0)$ & \\
\hline \multicolumn{3}{|l|}{ Cholangitis } \\
\hline$(0,0,4,0)$ & $(0,1,1,0)$ & \\
\hline \multicolumn{3}{|l|}{ Diarrhea } \\
\hline$(0,1,1,2)$ & $(0,1,3,2)$ & \\
\hline \multicolumn{3}{|l|}{ Nausea } \\
\hline$(0,0,1,0)$ & $(0,0,3,1)$ & \\
\hline \multicolumn{3}{|l|}{ Eczema } \\
\hline$(0,0,1,0)$ & $(0,0,1,1)$ & \\
\hline \multicolumn{3}{|l|}{ Heart failure } \\
\hline$(0,0,1,1)$ & $(0,1,0,0)$ & \\
\hline \multicolumn{3}{|c|}{ Interstitial pneumonia } \\
\hline$(0,0,0,1)$ & $(0,0,0,0)$ & \\
\hline \multicolumn{3}{|l|}{ Others } \\
\hline$(0,0,0,1)$ & $(0,0,2,0)$ & \\
\hline \multicolumn{3}{|c|}{ Discontinuation of adjuvant chemotherapy (Y: N) } \\
\hline 2: 19 & 9:9 & 0.011 \\
\hline
\end{tabular}

CTCAE Common Terminology Criteria for Adverse Events Boldface indicates the statistical significance

(GPS) was reported to be useful in predicting outcomes prior to adjuvant chemotherapy [21]. Aoyama et al. reported that $\mathrm{CCr}<60 \mathrm{~mL} / \mathrm{min}$ was a significant risk factor for the discontinuation of $\mathrm{S}-1$ adjuvant chemotherapy, even if renal function was normal according to serum creatinine levels [22]. In our study, we identified that a RDI $\geq 80 \%$ was significantly associated with a favorable prognosis. Additionally, we found that postoperative BWL $\geq 10 \%$ was associated with a reduced RDI $(<80 \%)$.

BWL correlates with lower quality of life and is the most reliable indicator of malnutrition. An association between BWL and poor prognosis has been reported in various cancers. Previously, Fearon et al. reported that unresectable pancreatic cancer patients with a BWL $\geq 10 \%$ had a lower objective function and worse prognosis [23]. Recently, Hashimoto et al. reported that severe postoperative BWL following pancreatectomy was significantly associated with poor prognosis [24]. Furthermore, a decrease in lean body mass could also affect the incidence of chemotherapy-induced toxicities. For gastric cancer patients who underwent curative resection, BWL $\geq 15 \%$ was the most important risk factor for S-1 adjuvant chemotherapy compliance [25]. In our study, BWL $\geq 10 \%$ tended to be related with poor prognosis and significantly influenced adjuvant chemotherapy continuity. Our study also emphasizes the importance of adequate perioperative nutrition in patients who receive adjuvant chemotherapy.

Recently, Sho et al. reported that elderly patients aged $\geq 80$ years were unlikely to complete the planned number of adjuvant chemotherapy cycles [26]. In our study, despite multiple logistic regression analysis not showing statistical significance, all patients $\geq 75$ years had discontinued or reduced (RDI < 80\%) adjuvant chemotherapy. Age is also a factor affecting adjuvant chemotherapy continuity for pancreatic cancer.

In this study, extended dissection of the nerve plexus around the SMA also had a negative effect on patient survival. Some randomized control trials have shown that extended lymphadenectomy, including dissection of the nerve plexus, has a minimal effect on patient survival. Farnell et al. reported that whole circumferential dissection of the nerve plexus around the SMA caused intractable diarrhea [27]. Recently, the International Study Group on Pancreatic Surgery (ISGPS) published a consensus statement regarding standard lymphadenectomy in surgery for pancreatic cancer. Complete resection around the SMA is not recommended [28]. BWL associated with diarrhea can cause nutritional and immunologic problems. Additionally, BWL may delay the initiation of postoperative chemotherapy.

This study has several potential limitations. The most significant one is that the results were derived from a retrospective single-center cohort with a small sample size. Next, the doses and intervals of the chemotherapy regimens varied among patients. With this taken into consideration, the results of this study should be verified in other large-scale series.

\section{Conclusions}

Our results indicate that patient with adjuvant chemotherapy of RDI $<80 \%$ significantly influence patient prognosis. BWL $\geq 10 \%$ after surgery is predictive of chemotherapy discontinuation. Adequate perioperative nutritional and surgical interventions are necessary for patients who receive adjuvant chemotherapy for advanced pancreatic cancer. However, a further prospective validation study is needed to confirm these findings, as the present study was retrospective and utilized a small sample size. 


\section{Abbreviations}

BSA: Body surface area; BWL: Body weight loss; CCr: Creatinine clearance; DFS: Disease-free survival; ESPAC: European Study Group for Pancreatic Cancer; GPS: Glasgow Prognostic Score; ISGPS: International Study Group on Pancreatic Surgery; JASPAC: Japan Adjuvant Study Group of Pancreatic Cancer; OS: Overall survival; RDI: Relative dose intensity; S-1: Tegafur/ gimeracil/oteracil; SMA: Superior mesenteric artery; SMV: Superior mesenteric vein

\section{Acknowledgements}

Not applicable

\section{Funding}

The authors received no specific funding for this work.

\section{Availability of data and materials}

The datasets used and/or analyzed during the current study are available from the corresponding author on reasonable request and approval of ethical committee of Hamamatsu University School of Medicine.

\section{Authors' contributions}

YM: design of the work, data acquisition, data analysis, drafting the work. TS: design of the work, drafting the work. RK (Ryo Kitajima): data acquisition, drafting the work. SF: data acquisition, drafting the work. RK (Ryota Kiuchi): data acquisition, drafting the work. MT: data acquisition, drafting the work. TH: data acquisition, drafting the work. YS: data acquisition, drafting the work. HK (Hirotoshi Kikuchi): drafting the work. HK (Hiroyuki Konno): drafting the work. HT: drafting the work. All authors read and approved the final manuscript.

\section{Ethics approval and consent to participate}

The study protocol conformed to the ethical guidelines of the 1975 Declaration of Helsinki and was approval by the ethical committee of Hamamatsu University School of Medicine (approval number: 15-318). Written consents to participate in this study were substituted for providing a means to opt out in the website (https://www.hama-med.ac.jp/research/clinical-res/erc/disclosure-info/h29.html) according to the ethics guidelines for clinical studies of the Japanese Ministry of Health, Labour and Welfare (MHLW)

\section{Consent for publication}

Not applicable

\section{Competing interests}

The authors declare that they have no competing interests.

\section{Publisher's Note}

Springer Nature remains neutral with regard to jurisdictional claims in published maps and institutional affiliations.

\section{Author details}

${ }^{1}$ Second Department of Surgery, Hamamatsu University School of Medicine, 1-20-1 Handayama, Higashi-ku, Hamamatsu 431-3192, Japan. ${ }^{2}$ Shizuoka city Shimizu hospital, Shizuoka, Japan. ${ }^{3}$ Hamamatsu University School of Medicine, Hamamatsu, Japan.

\section{Received: 22 January 2019 Accepted: 16 April 2019}

\section{Published online: 02 May 2019}

\section{References}

1. Bray F, Ferlay J, Soerjomataram I, Siegel RL, Torre LA, Jemal A. Global cancer statistics 2018: GLOBOCAN estimates of incidence and mortality worldwide for 36 cancers in 185 countries. CA Cancer J Clin. 2018;68(6):394-424.

2. Saad AM, Turk T, Al-Husseini MJ, Abdel-Rahman O. Trends in pancreatic adenocarcinoma incidence and mortality in the United States in the last four decades; a SEER-based study. BMC Cancer. 2018;18(1):688.

3. Siegel RL, Miller KD, Jemal A. Cancer statistics, 2018. CA Cancer J Clin. 2018; 68(1):7-30.

4. Krejs GJ. Pancreatic cancer: epidemiology and risk factors. Digestive diseases (Basel, Switzerland). 2010;28(2):355-8.

5. Ducreux M, Cuhna AS, Caramella C, Hollebecque A, Burtin P, Goere D, Seufferlein T, Haustermans K, Van Laethem JL, Conroy T, et al. Cancer of the pancreas: ESMO clinical practice guidelines for diagnosis, treatment and follow-up. Ann Oncol. 2015;26(Suppl 5):v56-68.

6. Tempero MA, Malafa MP, Al-Hawary M, Asbun H, Bain A, Behrman SW, Benson AB 3rd, Binder E, Cardin DB, Cha C, et al. Pancreatic adenocarcinoma, version 2.2017, NCCN clinical practice guidelines in oncology. J Natl Compr Canc Netw. 2017;15(8):1028-61.

7. Lim JE, Chien MW, Earle CC. Prognostic factors following curative resection for pancreatic adenocarcinoma: a population-based, linked database analysis of 396 patients. Ann Surg. 2003;237(1):74-85.

8. Xu X, Wu Q, Wang Z, Zheng S, Ge K, Jia C. Meta-analysis of FOLFIRINOX regimen as the first-line chemotherapy for locally advanced pancreatic cancer and borderline resectable pancreatic cancer. Clin Exp Med. 2019;19(1):149-57. https://doi.org/10.1007/s10238-018-0540-3. Epub 2018 Nov 29.

9. Peterson SL, Husnain M, Pollack T, Pimentel A, Loaiza-Bonilla A, WestendorfOverley C, Ratermann K, Anthony L, Desimone P, Goel G, et al. Neoadjuvant nab-paclitaxel and Gemcitabine in borderline Resectable or locally advanced Unresectable pancreatic adenocarcinoma in patients who are ineligible for FOLFIRINOX. Anticancer Res. 2018;38(7):4035-9.

10. Oettle H, Post S, Neuhaus P, Gellert K, Langrehr J, Ridwelski K, Schramm H, Fahlke J, Zuelke C, Burkart C, et al. Adjuvant chemotherapy with gemcitabine vs observation in patients undergoing curative-intent resection of pancreatic cancer: a randomized controlled trial. Jama. 2007;297(3):267-77.

11. Oettle H, Neuhaus P, Hochhaus A, Hartmann JT, Gellert K, Ridwelski K, Niedergethmann M, Zulke C, Fahlke J, Arning MB, et al. Adjuvant chemotherapy with gemcitabine and long-term outcomes among patients with resected pancreatic cancer: the CONKO-001 randomized trial. Jama. 2013;310(14):1473-81.

12. Uesaka K, Boku N, Fukutomi A, Okamura Y, Konishi M, Matsumoto I, Kaneoka Y, Shimizu Y, Nakamori S, Sakamoto H, et al. Adjuvant chemotherapy of S-1 versus gemcitabine for resected pancreatic cancer: a phase 3, open-label, randomised, non-inferiority trial (JASPAC 01). Lancet (London, England). 2016:388(10041):248-57.

13. Neoptolemos JP, Palmer DH, Ghaneh P, Psarelli EE, Valle JW, Halloran CM, Faluyi O, O'Reilly DA, Cunningham D, Wadsley J, et al. Comparison of adjuvant gemcitabine and capecitabine with gemcitabine monotherapy in patients with resected pancreatic cancer (ESPAC-4): a multicentre, open-label, randomised, phase 3 trial. Lancet (London, England). 2017;389(10073):1011-24.

14. Edge SB, Cancer AJCo: AJCC cancer staging handbook: from the AJCC cancer staging manual, vol. 2010: Springer New York; 2010.

15. Health UDo, Services $\mathrm{H}$ : Common terminology criteria for adverse events (CTCAE) version 4.0. National Institutes of Health, National Cancer Institute 2009, 4(03).

16. Cockcroft DW, Gault MH. Prediction of creatinine clearance from serum creatinine. Nephron. 1976;16(1):31-41.

17. Pawlik TM, Gleisner AL, Cameron JL, Winter JM, Assumpcao L, Lillemoe KD, Wolfgang C, Hruban RH, Schulick RD, Yeo CJ, et al. Prognostic relevance of lymph node ratio following pancreaticoduodenectomy for pancreatic cancer. Surgery. 2007;141(5):610-8.

18. Fujii T, Nakao A, Yamada S, Suenaga M, Hattori M, Takami H, Inokawa Y, Kanda $M$, Sugimoto $H$, Nomoto $S$, et al. Vein resections $>3 \mathrm{~cm}$ during pancreatectomy are associated with poor 1-year patency rates. Surgery. 2015;157(4):708-15.

19. Raut CP, Tseng JF, Sun CC, Wang H, Wolff RA, Crane CH, Hwang R, Vauthey $\mathrm{JN}$, Abdalla EK, Lee JE, et al. Impact of resection status on pattern of failure and survival after pancreaticoduodenectomy for pancreatic adenocarcinoma. Ann Surg. 2007:246(1):52-60.

20. Kondo N, Murakami Y, Uemura K, Hayashidani Y, Sudo T, Hashimoto Y, Nakashima A, Sakabe R, Shigemoto N, Kato $Y$, et al. Prognostic impact of perioperative serum CA 19-9 levels in patients with resectable pancreatic cancer. Ann Surg Oncol. 2010;17(9):2321-9.

21. Morinaga S, Murakawa M, Katayama Y, Yamaoku K, Aoyama T, Kanazawa A, Higuchi A, Shiozawa M, Kobayashi S, Ueno M, et al. Glasgow prognostic score predicts clinical outcomes in patients with pancreatic Cancer undergoing adjuvant gemcitabine monotherapy after curative surgery. Anticancer Res. 2015:35(9):4865-70.

22. Aoyama T, Katayama Y, Murakawa M, Asari M, Kanazawa A, Higuchi A, Shiozawa M, Kobayashi S, Ueno M, Morimoto M, et al. Risk factors for 6month continuation of S-1 adjuvant chemotherapy for resected pancreatic cancer. Cancer Chemother Pharmacol. 2014:74(6):1235-40.

23. Fearon KC, Voss AC, Hustead DS. Definition of cancer cachexia: effect of weight loss, reduced food intake, and systemic inflammation on functional status and prognosis. Am J Clin Nutr. 2006;83(6):1345-50. 
24. Hashimoto D, Chikamoto A, Ohmuraya M, Abe S, Nakagawa S, Beppu T, Takamori H, Hirota M, Baba H. Impact of postoperative weight loss on survival after resection for pancreatic Cancer. JPEN J Parenter Enteral Nutr. 2015;39(5):598-603.

25. Aoyama T, Yoshikawa T, Shirai J, Hayashi T, Yamada T, Tsuchida K, Hasegawa $\mathrm{S}$, Cho H, Yukawa N, Oshima T, et al. Body weight loss after surgery is an independent risk factor for continuation of S-1 adjuvant chemotherapy for gastric cancer. Ann Surg Oncol. 2013;20(6):2000-6.

26. Sho M, Murakami Y, Kawai M, Motoi F, Satoi S, Matsumoto I, Honda G, Uemura K, Yanagimoto $\mathrm{H}$, Kurata M, et al. Prognosis after surgical treatment for pancreatic cancer in patients aged 80 years or older: a multicenter study. J. Hepatobiliary Pancreat. Sci. 2016;23(3):188-97.

27. Farnell MB, Pearson RK, Sarr MG, DiMagno EP, Burgart $L$, Dahl TR, Foster N, Sargent DJ. A prospective randomized trial comparing standard pancreatoduodenectomy with pancreatoduodenectomy with extended lymphadenectomy in resectable pancreatic head adenocarcinoma. Surgery. 2005:138(4):618-28 discussion 628-630.

28. Tol JA, Gouma DJ, Bassi C, Dervenis C, Montorsi M, Adham M, AndrenSandberg A, Asbun HJ, Bockhorn M, Buchler MW, et al. Definition of a standard lymphadenectomy in surgery for pancreatic ductal adenocarcinoma: a consensus statement by the international study group on pancreatic surgery (ISGPS). Surgery. 2014;156(3):591-600.

Ready to submit your research? Choose BMC and benefit from:

- fast, convenient online submission

- thorough peer review by experienced researchers in your field

- rapid publication on acceptance

- support for research data, including large and complex data types

- gold Open Access which fosters wider collaboration and increased citations

- maximum visibility for your research: over $100 \mathrm{M}$ website views per year

At $\mathrm{BMC}$, research is always in progress.

Learn more biomedcentral.com/submissions 